\title{
Growing concerns for COP26
}

\author{
Anthropogenic climate change is often portrayed as a consequence of industrialization powered by fossil fuel \\ consumption. However, agriculture also plays a role in this complex system of causes and effects.
}

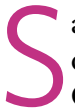
aturday 30 October will see the opening of the COP26 Climate Change Conference in Glasgow, UK. COP stands for the Conference of the Parties and is the governing body of any international convention. As such, there are many COPs for the many international conventions. This particular 'COP' relates to the United Nations Framework Convention on Climate Change (UNFCCC), although the importance of this convention has made defining that redundant. To most people, COP means climate change.

These meetings have a long history. The UNFCCC was originally signed by 154 states at the United Nations Conference on Environment and Development (UNCED) held in Rio de Janeiro in 1992; commonly known as the Rio Earth Summit. The first UNFCCC COP was held in Bonn, Germany in 1995 and these meetings have occurred essentially annually ever since.

Some COPs have been more significant than others. The third COP (COP3), held in Kyoto, Japan in 1997, saw the negotiation and adoption of the Kyoto Protocol, which set out legally binding commitments from its signatories to reduce their emissions of greenhouse gases over the period 2008-2012. This was amended at the COP of 2012 (COP18) in Doha, Qatar to extend up to 2020.

COP21, held in Paris, France in 2015, produced an agreement designed to take over from the Kyoto Protocol when it expired in 2020, with both an intent to hold average global temperatures to less than $2{ }^{\circ} \mathrm{C}$ above pre-industrial levels and a commitment from states to increase their individual targets for greenhouse gas emissions every five years. COP26, which should have been held in 2020 but was delayed because of the COVID-19 pandemic, will be the first Conference since the Paris Agreement took effect and will provide a clear indication of how far behind its aims we are falling.
Carbon dioxide is the archetypal greenhouse gas, whose release into the atmosphere results in the raising of global temperatures. As a raw material of photosynthesis, this puts plants and associated photosynthetic organisms at the centre of any discussion of the causes and responses to climate change. It is not unreasonable to regard the release of $\mathrm{CO}_{2}$ into the atmosphere by the burning of oil, gas, and particularly coal over the last several hundred years as reversing the work done by plants during the carboniferous era (360 to 300 million years ago) to 'lock up' carbon underground. During that period, global average temperatures dropped from around $20^{\circ} \mathrm{C}$ to $12^{\circ} \mathrm{C}$, and atmospheric $\mathrm{CO}_{2}$ dropped approximately eightfold.

This view of plants as carbon sinks can lead to an assumption that agricultureespecially the growing of crop plantsshould be a reducer of greenhouse gas emissions, or at least 'carbon-neutral'. The true situation is not so simple. A recent paper in Nature Food (Crippa, M. et al., Nat. Food 2, 198-209; 2021) audited the greenhouse gas emissions from food systems and found that they accounted for 18 gigatonnes of $\mathrm{CO}_{2}$ equivalents in 2015 (the most recent year in their analysis), and that most of this occurred before food left the farm. That represents about a third of global greenhouse gas emissions. Much of this is related to land use changes, but there are other important contributions. Another Nature Food study (Xu, X. et al., Nat. Food 2, 724-732; 2021) performed a similar audit, the main conclusion of which was that agriculture for animal-based food emitted twice as much $\mathrm{CO}_{2}$ as for purely plant-based foods. The authors also identified 0.7 gigatonnes of $\mathrm{CO}_{2}$ equivalents as arising from the production of inputs such as fertilizers.

That artificial fertilizers should be such a substantial part of modern agriculture should be no surprise. In the nineteenth century and earlier, guano (sea-bird faeces) was a highly prized commodity owing to its nitrogen content. In the 1860s, Spain and Peru went to war over the former's occupation of the guano-rich Chincha Islands, a conflict that soon involved Equador, Chile and Bolivia as well. Far earlier than this, Chile's Atacama desert was a productive area of agriculture thanks to the establishment of a transport route to bring the nitrogenous 'white gold' up from the coast (Santana-Sagredo, F. et al., Nat. Plants 7, 152-158; 2021). Reliance on guano for nitrogenous fertilizer was brought to an end in the early twentieth century by the discovery, by the German chemist Fritz Haber, of a method to synthesize ammonia from atmospheric nitrogen and hydrogen at high temperatures and pressures, with the use of a metal catalyst. This process was industrialized by another German, Carl Bosch, and the Haber-Bosch process remains the main route for industrial nitrogen fixation more than a hundred years later. The process requires a great deal of energy. It has been calculated that the powering of the Haber-Bosch process alone accounts for $1 \%$ of global $\mathrm{CO}_{2}$ emissions, so alternative methods of synthesis are actively being sought (Ashida, Y. et al., Nature 568, 536-540; 2019).

Tackling climate change is a complex problem that requires a diversity of approaches (as is amply demonstrated by the range of studies included in a Nature Collection recently assembled in advance of COP26). Developments in agriculture will be crucial to any successful solution, whether involving plant breeding (Xiong, W. et al., Nat. Plants 7, 1207-1212; 2021) or other technologies (Horton, P. et al. Nat. Plants 7, 250-255; 2021).

Published online: 14 October 2021 https://doi.org/10.1038/s41477-021-01012-X 